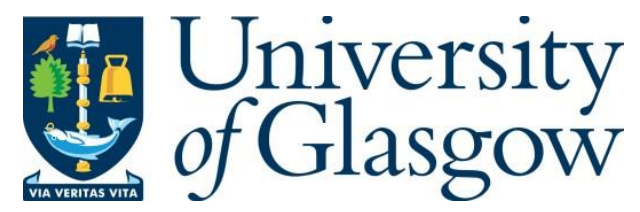

Li, G., Sarma, J. and Hogg, R. A. (2018) Modal Index Analysis of Resonances of PCSEL. In: 2018 International Conference on Numerical Simulation of Optoelectronic Devices (NUSOD), Hong Kong, China, 05-09 Nov 2018, pp. 131-132. ISBN 9781538655993.

There may be differences between this version and the published version. You are advised to consult the publisher's version if you wish to cite from it.

http://eprints.gla.ac.uk/182532/

Deposited on: 22 March 2019

Enlighten - Research publications by members of the University of Glasgow http://eprints.gla.ac.uk 


\title{
Modal Index Analysis of Resonances of PCSEL
}

\author{
Guangrui $\mathrm{Li}^{1}$, Jayanta Sarma ${ }^{1}$, Richard A. Hogg ${ }^{1}$ \\ ${ }^{1}$ School of Engineering, the University of Glasgow, Rankine Building, Glasgow, G12 8LT, United Kingdom \\ e-mail: g.li.2@research.gla.ac.uk
}

\begin{abstract}
This paper presents a new modal index analysis method for evaluating the resonances of PCSEL structures which is versatile, efficient and fast. Hence it is envisaged that the implementation of this method will enhance the potential to generate more comprehensive models of photonic crystal based devices, say, PCSELs, that include, for example, aspects of inversion population distribution and also time dependence while still retaining relatively modest demands on computational resources.
\end{abstract}

\section{INTRODUCTION}

The photonic crystal surface emitting laser (PCSEL) has shown significant promise recently and has received much attention because of its potential to generate high power, monochromic operation with a narrow output beam from a large optically active area [1]. A primary requirement in modelling PCSELs is the evaluation of the optical field distribution that identify the resonant (lasing) mode. Conventional techniques such as plane wave expansion (PWE)[2], finite difference time domain (FDTD)[3], and coupled mode theory (CMT) [4] have been widely used to analyse the pertinent 2-D photonic crystal (PC) structures. However, these techniques are either computationally very time consuming or mathematically rather intensive and demanding.

In this paper, an alternative approach based on modal indices analysis (MIA) which is applicable to a piecewise constant media, in combination with a simple and fast numerical computation is used to evaluate the resonant modes in a rectangular geometry 2-D PC structure.

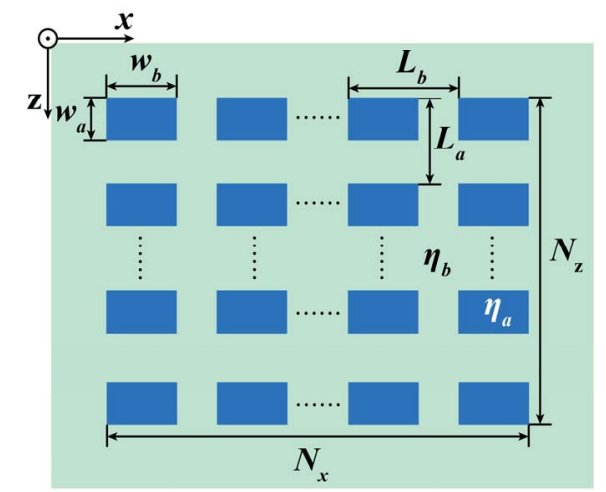

Fig. 1 Schematic of PC structure. $N_{x}$ and $N_{\mathrm{z}}$ are number of periods along $x$ and $\mathrm{z}$ respectively.

\section{MODEL DESCRIPTION}

A rectangular co-ordinate system $(x, y, z)$ is used consistent with the rectangular device geometry. Fig. [1] which gives a schematic representation of the pertinent planar periodic structure in the $x$-z plane with a refractive index profile $\eta(x, z)$, and the excitation is taken to be such that any non-zero field component, $\hat{F}(x, y, z)=\hat{F}(x, z)$ [5], i.e., $\partial / \partial y \approx 0$ is applicable. Any nonzero field component $\hat{F}_{m, n}(x, z)$ for the resonance modes ( $m, n$; integers) satisfied the wave equation:

$$
\left[\frac{\partial^{2}}{\partial x^{2}}+\frac{\partial^{2}}{\partial z^{2}}+k_{0}^{2} \eta^{2}(x, z)\right] \hat{F}(x, z)=0
$$
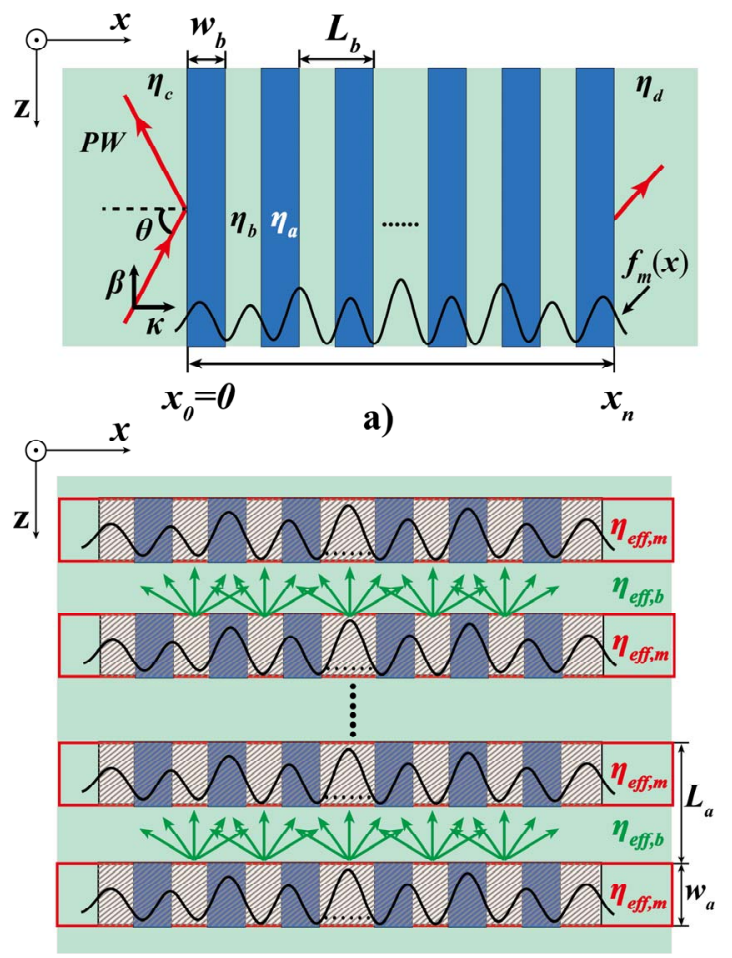

b)

Fig. 2 Model description: a) Lateral modes propagating along the zaxis are computed for a relevant range of wavelengths. b) The shaded area with dark blue region representing the multilayer waveguide in Fig. 2a) is replaced by a homogeneous medium of effective modal index $\eta_{\text {eff }, m}$, thus resulting in a 1-D periodic grating along $\mathrm{z}$ with a unit cell composited of $\eta_{e f f, m}$ and $\eta_{e f f, b}$.

The procedure for obtaining $\hat{F}_{m, n}(x, z)$ begins by first considering a piecewise-constant multilayer structure as shown in Fig.[2a]; the characteristic waves (modes), $f_{m}(x)$ which propagates along the $\mathrm{z}$-axis with the corresponding mode indices $\eta_{\text {eff, } m}$ are evaluated over a pertinent range of wavelength using the transfer matrix method[6] as an excitation problem rather than as an eigenvalue problem[7] as the former readily evaluates a wider range of solutions and provides more insight. 


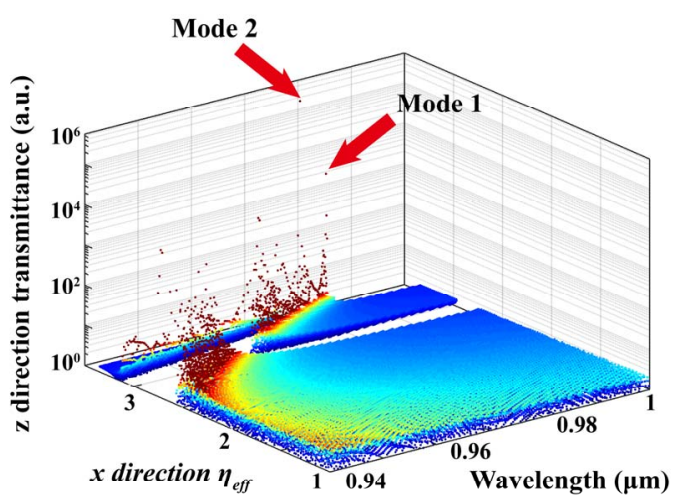

a)

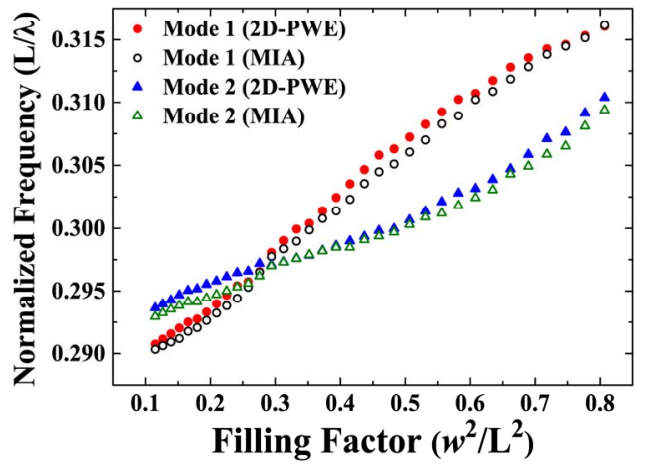

b)

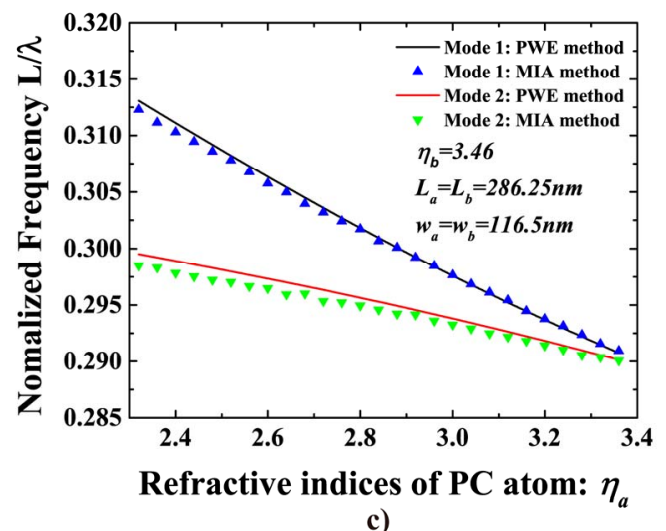

Fig. 3 a) 2-D resonances of PC calculated using MIA. (band edge resonances: Mode 1 and Mode 2). b) Band edge resonances calculated with varying filling factor. c) Band edge resonances calculated with varying $\eta_{a}$

Then, discontinuities along the z-axis are introduced in to the (waveguide) structure, Fig. [2a] to 'create' the 2-D PC structure corresponding to Fig. [1] and this is then analysed as a waveguide discontinuity problem. Multimode generation at discontinuities [8] is approximated by single (self-mode) conditions so that a simple modal index (mode impedance) analysis at discontinuities is used. However, importantly, consideration of diffraction of modes in to the homogenous region $\left(\eta_{b}\right)$ at the discontinuities provides a modified (effective) index for the homogenous regions. Thus, the refractive indices in segmented regions (as shown in Fig. [2b] with green arrows) are further modified accordingly into $\eta_{\text {eff }, b}$ using in-plane diffraction[9]. The transfer matrix method is then used to obtain the resonances $g_{n, m}\left[z ; f_{m}(x)\right]$ of such effective periodic structure (Fig. [2b]) composited of unit cell $\left\{\eta_{e f f, m}, \eta_{e f f, b}\right\}$, which in effect, includes reasonably well the characteristics of the original 2-D periodic media (Fig. [1]). Hence the final 2-D field distribution at resonances are $\hat{F}_{m, n}(x, z)=f_{m}(x) g_{n, m}\left[z ; f_{m}(x)\right]$.

\section{RESULTS AND DISCUSSIONS}

The resonance wavelength is searched over an appropriate range and the final result (resonances), $\hat{F}_{m, n}(x, z)$, of such structure are represented by $\left(\eta_{e f f, m}, \lambda_{0}\right)$ pairs, referring to Fig. [3a]. Optical gain are included as a convenience for easier identifying band edge (lasing) mode as illustrated in Fig. [3a]. Band edge modes obtained by this method match closely with those calculated using CMT over a large range of filling factor (Fig. [3b]) and over a large range of refractive index differences (Fig. [3c]).

Further detail of modelling process, analysis of finite size PCs, with discussions on the consistency and validity of the model will be presented at the conference.

\section{CONCLUSION}

In this work, a novel method for solving resonance in 2-D PC using mode index analysis is presented. The method is based on wave propagation in periodic multilayer structure. Full use of transfer matrix makes the computation process modest and fast. It is shown that the method is versatile and yields very reliable results. In view of above, the MIA method is promising in more comprehensive device modelling such as spatial and temporal variation in optical gain and other PC configuration.

\section{REFERENCES}

[1]. K. Hirose, Y. Liang, Y. Kurosaka, A. Watanabe, T. Sugiyama, and S. Noda, "Watt-class high-power, high-beam-quality photonic-crystal lasers," Nat. Photonics, vol. 8, no. 5, pp. 406-411, 2014.

[2]. M. Plihal and A. A. Maradudin, "Photonic band structure of twodimensional systems: The triangular lattice," Phys. Rev. B, vol. 44, no. 16, pp. 8565-8571, Oct. 1991.

[3]. S. Fan and J. Joannopoulos, "Analysis of guided resonances in photonic crystal slabs,” Phys. Rev. B, vol. 65, no. 23, pp. 1-8, 2002.

[4]. I. Vurgaftman and J. R. Meyer, "Design optimization for high-brightness surface-emitting photonic-crystal distributee-feedback lasers," IEEE J. Quantum Electron., vol. 39, no. 6, pp. 689-700, 2003.

[5]. H. Han and J. J. Coleman, "Two-dimensional rectangular lattice distributed feedback lasers: A coupled-mode analysis of TE guided modes," IEEE J. Quantum Electron., vol. 31, no. 11, pp. 1947-1954, 1995.

[6]. A. Ghatak, K. Thyagarajan, and M. Shenoy, "Numerical analysis of planar optical waveguides using matrix approach," J. Light. Technol., vol. 5, no. 5, pp. 660-667, 1987.

[7]. D. J. Griffiths and C. A. Steinke, "Waves in locally periodic media," Am. J. Phys., vol. 69, no. 2, p. 137, 2001.

[8]. T. E. Rozzi, "Rigorous Analysis of the Step Discontinuity in a Planar Dielectric Waveguide," in IEEE Transactions on Microwave Theory and Techniques, vol. 26, no. 10, pp. 738-746, Oct 1978.

[9]. Akira Ishimaru, Electromagnetic Wave Propagation, Radiation, and Scattering:From Fundamentals to Applications, Wiley-IEEE Press, 2017. 